\title{
Tailored Rislk Management In Drilling \& Completion: Illustrative Case Study Of A Well Campaign
}

\author{
F. Zausa, S. Masi, C. Repetto, eni e\&p, J. Michelez, N. Rossi, kwantis
}

\section{$\underline{\text { Abstract }}$}

\section{Risk Management (RM) is a}

well recognised approach to address projects uncertainties, to provide accurate CAPEX estimates and to have proactive responses to unplanned events.

However, RM is often presented as a standard and repetitive process, disregard to projects diverse challenges. This acceptance is a main obstacle to its successful implementation, because this can lead to over complex risk studies for simple matter or, on the contrary, scarce results for intricate issues.

Drilling \& Completion projects present a high versatility of situations (from a standard onshore workover to a very challenging deep offshore exploratory campaign). Project challenges can be very different: technical (HP/HT, new technologies, etc), environmental (sensitive areas, deep offshore conditions, etc), economic (market conditions, etc), or strategic (partnership, etc).

The natural consequence is that each project requires an adequate response in terms of RM. Actually, RM toolbox encompasses a number of different studies, such as risk registers, risk mapping, probabilistic CAPEX/schedule, fault trees, etc.

Not all studies should be systematically performed. Thus, the $\mathrm{RM}$ approach must be tailored to each project challenges, in order to bring the highest added value to the decision makers. 
eni Drilling, Completion \& Production optimization department has built an integrated RM system that provides a properly designed workflow for treating each project with a suitable approach according to its maturity and other technical, environmental, economic or strategic criteria.

This paper takes advantage of an eni experience on a well campaign and describes how RM approach supported strategic decisions on the implementation of technologies (RSS, continuous circulating system, aluminium drill pipe, ceramic protectors and centralisers) to reduce well operations uncertainties. This case study encompasses the complete decision loop, from the cost/benefit analysis of each technology to the measured benefits from the rig operations in terms of performance enhancement.

Finally, it substantiates the values of $\mathrm{RM}$ in investment decision by addressing $\mathrm{RM}$ in a practical way. This case study will definitely help to spread a RM culture within project management organizations.

\section{$\underline{\text { Introduction }}$}

The aim of this paper is to present the added value of project Risk Management (RM) activities applied to Drilling \& Completion (D\&C) activities.

\section{$\underline{\text { Project Risk Management }}$}

By definition, a Project Risk is "an uncertain event or condition that, if it occurs, has a positive or a negative effect on at least one project objective. A risk may have one or more causes and, if it occurs, one or more impacts" 1. Project objectives are multiple and are usually addressed by complementary approaches:

- HSE (Health, Safety \& Environment) actively monitor any potential risk issue regarding safety, 
security or environment

objectives

- RM (Risk Management) actively monitor any potential risk issue regarding economic objectives; mainly schedule, CAPEX, OPEX, revenues and corporate relations with third parties.

Project risk management covers a number of techniques and tools that are adapted to any specific problematic; i.e. calculation of a complex event probability 2 , risk mapping, probabilistic cost estimates,...

\section{Drilling And Completion Projects}

D\&C activities are known as highly challenging activities for many reasons, such as:

- Technical complexity

- Multiple sub-systems

- Continuous innovations

- High reliability requirements

- High constraints on cost and time
- High operation costs and limited margin for overrun

- Large CAPEX

- Difficult budget accuracy

- Project Organization

- Numerous contractors to be coordinated

- Different cultures, perspectives and objectives of the participants

- Logistical constraints

- Lack of historical feedback

- Market conditions

- Highly uncertain conditions about commodities, services costs, ..

- Payment in different currencies

Also D\&C projects represent a high versatility of situations, e.g. a standard onshore work over versus a complex deep offshore campaign. 
RM activities must respond to the complexity and the challenges of each project with the most adequate level of resources.

\section{Risk Management Ojectives And}

\section{$\underline{\text { Key Principles }}$}

\section{When implementing its}

$\mathrm{RM}$ process, eni has considered a number of key principles that would emphasize the added value of the system. These key principles are distributed between two project management aspects: methodological (estimates accuracy) and organizational (data integration).

\section{Estimates Accuracy}

$$
\text { It is a difficult exercise to }
$$

perform time and cost estimates on $\mathrm{D} \& \mathrm{C}$ projects, due to the versatility of the projects and the number of external factors that can influence project success (weather, lithology, political stability,...). For that reason cost overrun and project delays are a 


\section{EXPERTISE \\ engineering \\ LESSONS LEARNED \\ cost control, post drill analysis,... \\ UNCERTAINTIES \\ project risk management \\ REFERENCES \\ offset wells, contracts, previous projects,...}

Fig.(1) Cost \& Time Estimates Accuracy 4 Pillars

$\underline{\text { References are the most straight }}$

forward source of information. They are built on any similar technical, local or contractual situations. In that case future project estimates shall reflect performance indicators or Non-Productive Time (NPT) statistical records. Limitations for project references are associated with data reliability and applicability. Data reliability depends on database availability, therefore it is necessary to have a sufficient level of records detail and good quality data (typically some unplanned or NPT could be covered in planned activities). Data applicability is related to the relevancy of the statistical records for the project being studied. Before being used, statistical records deserve a critical lecture to evaluate whether technical progress, contractor changes or local conditions are limiting their relevance.

\section{$\underline{\text { Uncertainties }}$ are inherent to} project management, as the future is uncertain. As a consequence zero risk is not realistic and there is no project without any risk. However uncertainties can be acceptable for a project when they are well understood, managed, allocated to 
the most competent party and controlled. This is the role of RM.

\section{Lessons Learned is necessary to} close the loop in project management. All projects should be reassessed during and after their life in order to measure how accurate estimations have been done. Besides, this also helps to understand potential discrepancies between planned and actual data and to implement the corrective actions in a positive sense of continuous learning.

$\underline{\text { Expertise }}$ is the essence of project management, since no tool or system will ever be able to address the complexity of a new venture. So engineering, analysis and verifications are necessary at every step of a time and cost process.

\section{Data Integration}

Major O\&G corporate are giant companies dealing with a number of affiliates, personnel mobility and restraint information for confidentiality matters. This situation leads to a dilution or a loss information that could be crucial for project management.

To face that situation, most project activities are supported by integrated systems, to organise and share project data.

Regarding RM activities the solution is to implement an integrated system that will provide a:

- Standardization of the workflow and results according to company procedures and each project characteristics.

- Assistance to any contributor in performing qualitative and quantitative risk analyses and a monitoring of the precision 
and completeness of these studies.

- Traceability of information from feasibility studies to project execution.

- Organization of the RM activities and sharing of the information among all contributors with the proper level of confidentiality and safety.

The system implemented for RM applied on D\&C projects in eni is named AWARE for Advised Workflow for Accurate Risk Estimates and is organised as pictured below:

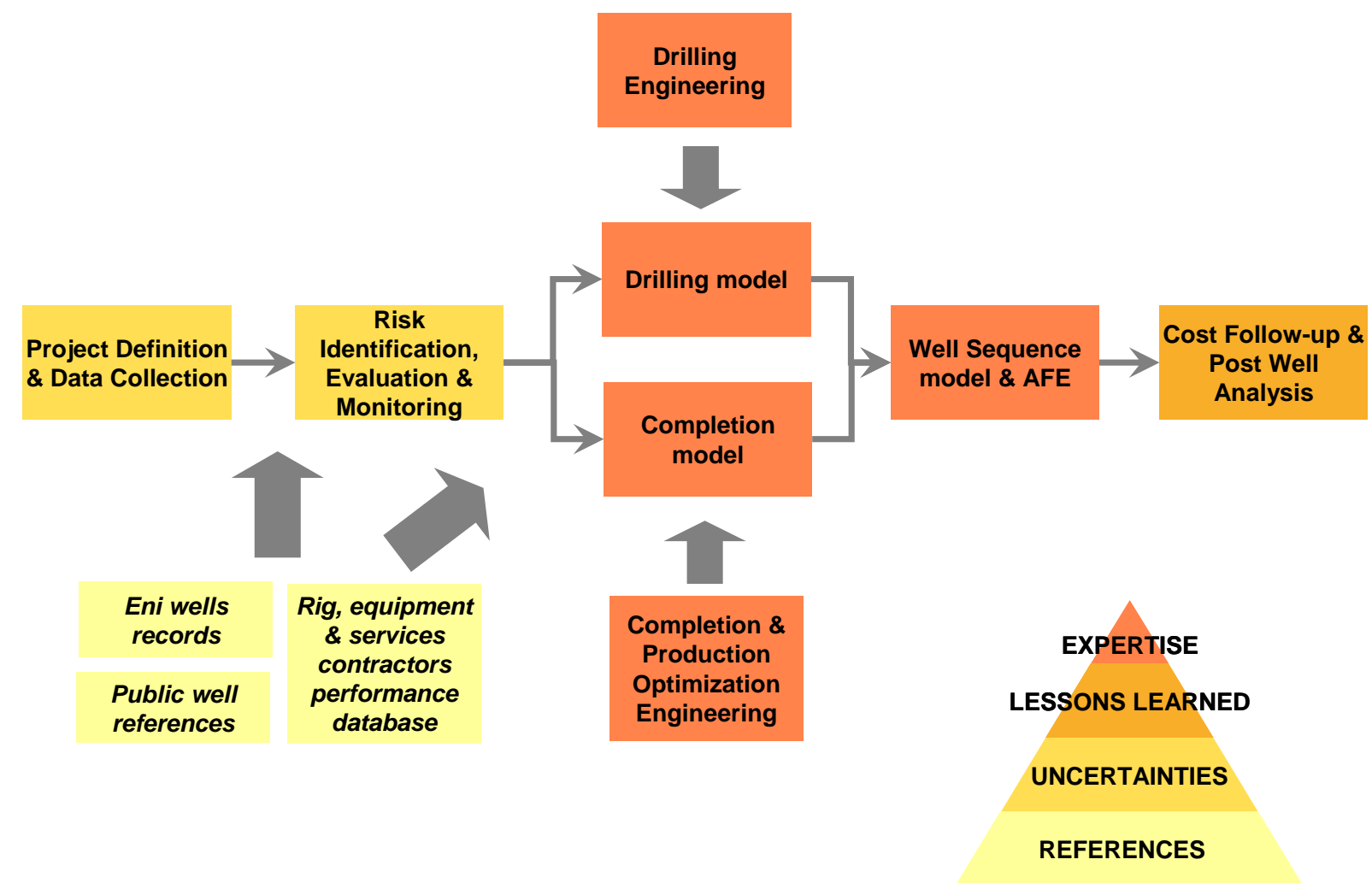

Fig.(2) RM Integrated Data System

The system is a platform that has a number of specific applications and linked databases (well records, probabilistic application, risk register,...). User access \& rights are managed through project groups.

All 4 estimates pillars are covered through the process. Basically any 
relevant well references is used either for risk identification or for probabilistic variables definition. All information regarding uncertainties definition and monitoring is gathered in a risk register. Qualitative risk evaluation is done through matrixes. Expertise is present at every step of the process and more specifically to build the probabilistic model that will bring the AFE (Authorization for Expenditure), either for one single well of a multiple well D\&C sequence. Lessons Learned are built up from actual project execution data and confronted with the AFE estimates. An illustration of this workflow is presented in the following chapter.

\section{Methodology}

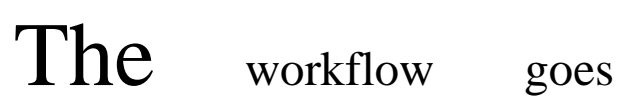

through a series of 10 steps and 2 potential optimization loops as presented in the below diagram:

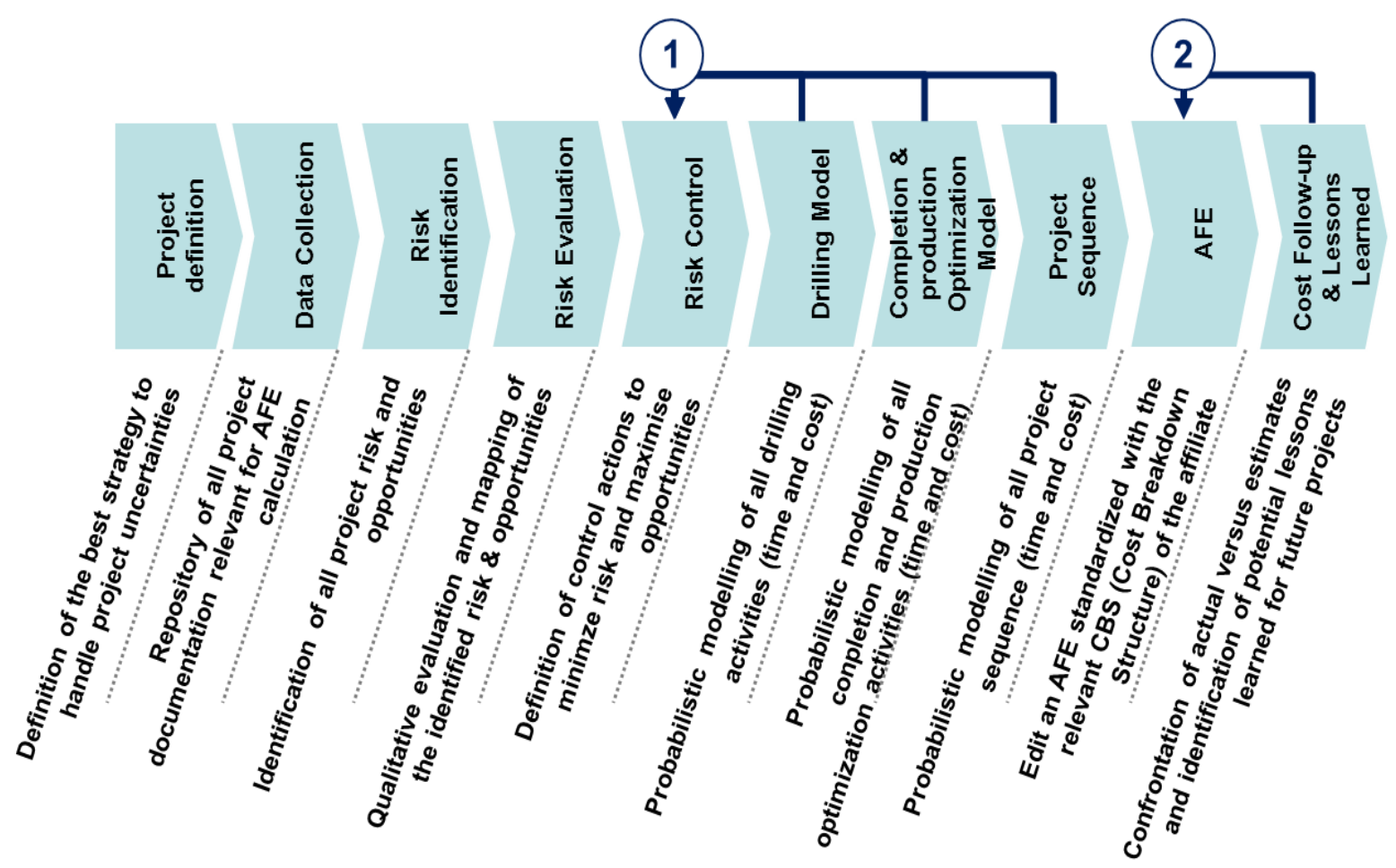

Fig.( 3) RM Workflow 
The 10 steps are performed through the guided interface of the AWARE system. The optimization loops are iterations in the workflow in order to reduce the uncertainties and to enhance the accuracy of AFE estimates:

\section{1}

At any stage of the probabilistic modelling, it is useful to measure the efficiency of the risk control actions in terms of costs versus benefits.

2

During project execution, a potential time or cost overrun exceeding the contingencies provided for the project, might require a re-authorization of the project. In that situation a time and cost re-evaluation is performed on the probabilistic modelling.

\section{Case Study}

\section{The case study is presented}

through all the steps above described. In this specific example, the scope of the project is limited to the Drilling activities of one
$1^{\text {st }}$ Iraq Oil \& Gas Conference $\left(1^{\mathrm{ST}}\right.$ IOGC $)$ extended reach well. The risk study was performed in 2009 and the well was drilled in the early of 2010 . Some names and data in this paper have been changed in order to guarantee the confidentiality of the project, nonetheless without altering real principles and conclusions.

\section{$\underline{\text { Project Definition }}$}

The first step of the RM process starts with a definition of the project in order to bring a complete picture of project scope and challenges. From this step, a series of tips and recommendations are given by AWARE to perform all RM analyses. For example, in this case study the system guide the user towards probabilistic modelling based on reference wells data, suggest a series of typical risk issues to be considered in ERD wells,...

The well under study is a challenging ERD well. A high number of NPT was experienced on the first 3 wells that have been drilled. NPT were caused by several 
reasons such as hole quality and well control related issues, and they were not limited to equipment reliability.

This leaded up to $100 \%$ cost overrun on some wells therefore requiring an urgent evaluation of potential mitigation measures.
Well profiles main characteristics are presented in the below table:

Table(1) Well profile

\begin{tabular}{|c|c|c|c|c|c|c|}
\hline \multirow{2}{*}{ Phases } & \multicolumn{2}{|c|}{ Drilling } & \multicolumn{2}{|c|}{ Casing } & \multirow{2}{*}{$\begin{array}{l}\text { TVD } \\
\text { (m) }\end{array}$} & \multirow{2}{*}{ Incl } \\
\hline & $\begin{array}{c}\mathrm{OH} \\
\text { (MD) }\end{array}$ & $\begin{array}{l}\text { Lengths } \\
\text { (MD) }\end{array}$ & Susp & $\begin{array}{l}\text { Lengths } \\
\text { (MD) }\end{array}$ & & \\
\hline $\begin{array}{c}26 " \text { hole/ } \\
20 " \text { csg }\end{array}$ & 350 & 350 & -150 & 500 & & \\
\hline $\begin{array}{c}16 \text { " holel } \\
13 \text { 3/8" csg }\end{array}$ & 1850 & 1500 & -150 & 2000 & 1400 & $60^{\circ}$ \\
\hline $\begin{array}{c}12 \text { 1/4" hole } 1 \\
95 / 8 " \mathrm{csg}\end{array}$ & 5850 & 4000 & -150 & 6000 & 2000 & $80^{\circ}$ \\
\hline $\begin{array}{c}81 / 2 " \text { hole } / \\
7 " \mathrm{nr}\end{array}$ & 7400 & 1550 & 5750 & 1650 & 2250 & $90^{\circ}$ \\
\hline 6" hole & 8000 & 600 & & & 2250 & \\
\hline
\end{tabular}

\section{$\underline{\text { Data Collection }}$}

This step is performed

through the collection of project main documentation (technical, contractual, organizational, etc.). In this specific case, many offset data were available from 3 existing and comparable wells already drilled. Offset well data analysis was both used for risk identification and risk evaluation. 


\section{$\underline{\text { Risk Identification }}$}

$\mathbf{R}_{\text {isk identification involves }}$ identifying all the potential risks associated with the project objectives (in this specific case drilling time and cost). 14 risks were identified (see table 0 Risk Allocation of the next section) through 3 sources:

- Offset well analysis

- Experts interviews

- Risk check list

\section{$\underline{\text { Risk Evaluation (Qualitative) }}$}

$\mathbf{R}_{\text {isk evaluation is qualitative }}$ in order to provide a high level of understanding and prioritization of the risks of the project. Risk assessment requires each risk to be estimated according to its probability and impact (time and or cost). The qualitative evaluation brings to a ranking of the risk that is classified as Low, High, Medium, as per table 0any of these probability and impacts can be later built into the probabilistic model during the quantitative evaluation.

\section{$\underline{\text { Risk Control }}$}

After risk identification and evaluation, the logical successive step is to propose / assess some control actions to minimise risk impacts to a reasonable level. In this case 5 different actions were proposed to mitigate the most critical risk issues, either as management or technological actions.

\section{Project management:}

- Engineering: further studies for trajectory optimization

- Training: specific crew training for ERD wells drilling

\section{Technologies implementation:}

- Rotary Steerable System (RSS) helps to avoid excessive dog legs and hole 
tortuosity when drilling vertical, deviated and horizontal wells.

- Eni Circulation Device (ECD) that allows uninterrupted mud circulation while breaking out or making up drill pipe connections during drilling operation. This implies good control of the Equivalent

Circulation

Density as it avoids any pressure fluctuation; it helps to reduce NPT, to improve borehole stability, to diminish drilling time and costs and also it helps to reach inaccessible reservoirs.

- Aluminium Drill Pipes equipped with ceramic protectors (ADP) that are the right solution for ERD wells as they are more flexible than conventional drill pipes; they have higher corrosion resistance and excellent fatigue resistance; they also contribute to reduce friction factors.

\section{Drilling Model}

The drilling model is a computation of the drilling program time and cost sequence combined with the probability and consequence of all individual risks.

The result is giving a global picture of the project uncertainties and more specifically the probability that the project will meet quantitative objectives (schedule and budget), and the definition of the related level of contingencies to be considered.

Each risk was numerically integrated in the drilling operational sequence, with its related probability and cost and/or time impact. Risk allocation is illustrated in the below table: 
Table(2) Risk Allocation

\begin{tabular}{|c|c|c|c|c|c|c|c|c|}
\hline $\mathbf{N b}$ & Risk & 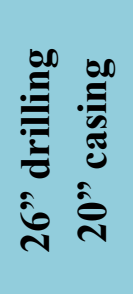 & 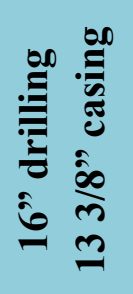 & 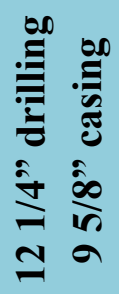 & 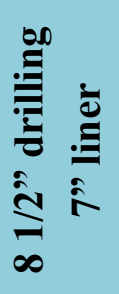 & 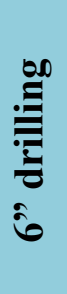 & 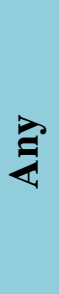 & 焉 \\
\hline 1 & Kick/Well control & & & & & & & $\mathrm{L}$ \\
\hline 2 & $\begin{array}{l}\text { Well Collision/Close } \\
\text { trajectories }\end{array}$ & & & & & & & L \\
\hline 3 & $\begin{array}{l}\text { Wait due to } \\
\text { spills/environmental } \\
\text { issues }\end{array}$ & & & & & & & $\mathrm{L}$ \\
\hline 4 & $\begin{array}{l}\text { Twist off/Stuck } \\
\text { pipe/Fishing }\end{array}$ & & & & & & & L \\
\hline 5 & $\begin{array}{l}\text { BOP equipment } \\
\text { problems }\end{array}$ & & & & & & & $L$ \\
\hline 6 & Casing wear & & & & & & & $M$ \\
\hline 7 & Sidetrack & & & & & & & $\bar{M}$ \\
\hline 8 & Stuck casing & & & & & & & $\bar{M}$ \\
\hline 9 & $\begin{array}{l}\text { Cement unit/job } \\
\text { problems }\end{array}$ & & & & & & & $\mathrm{L}$ \\
\hline 10 & $\begin{array}{l}\text { MWD/LWD/BHA } \\
\text { failure }\end{array}$ & & & & & & & M \\
\hline 11 & $\begin{array}{l}\text { Wait on rig repair/tool } \\
\text { repair or replacement }\end{array}$ & & & & & & & M \\
\hline 12 & $\begin{array}{l}\text { Hole cleaning } \\
\text { problems }\end{array}$ & & & & & & & $\mathrm{H}$ \\
\hline 13 & Mud losses & & & & & & & $\mathrm{L}$ \\
\hline 14 & Wait on weather & & & & & & & $\mathrm{M}$ \\
\hline
\end{tabular}

A Monte Carlo Simulation Was Run Giving The Following Results On The Base Case: 


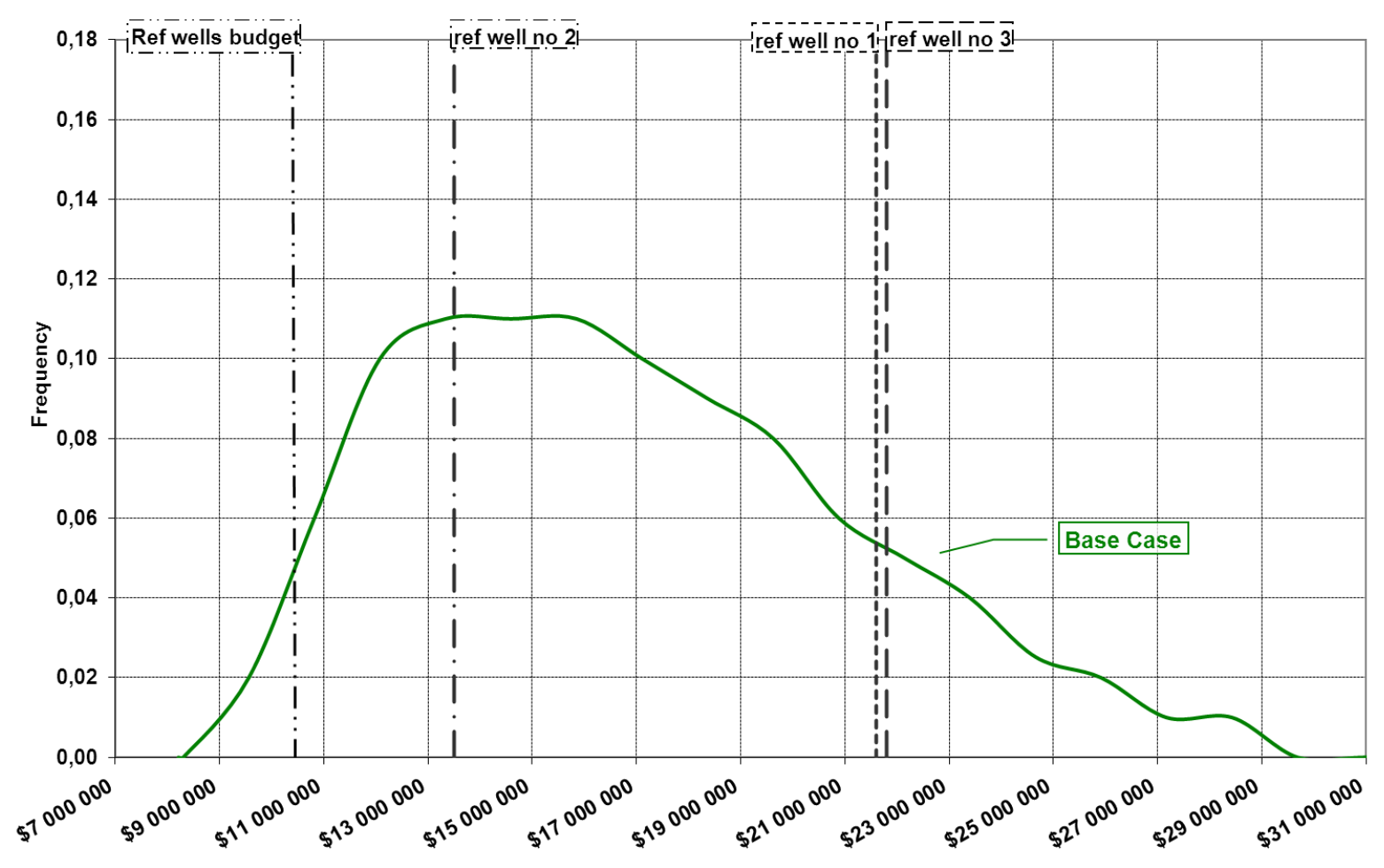

Fig.(4) Base Case Probabilistic Cost

This graph shows the cost distribution without implementing any mitigation action. Furthermore, the 3 available reference wells that were previously drilled with their related budget are also represented in the plot. It shows that budget underestimated uncertainties leads to major cost overrun.

\section{Drilling Model Optimization}

In order to evaluate the cost and benefits of the proposed control actions, the model was run through 4 different scenarios:

- Base Case $=$ no mitigation action

- Scenario A = Base Case + Engineering + RSS in 16"

- Scenario B = Scenario A + E-CD + Training

- Scenario C = Scenario B + Aluminium DP 
The cost/benefits impact of the optimized scenarios is presented in the below graph along with the base case:

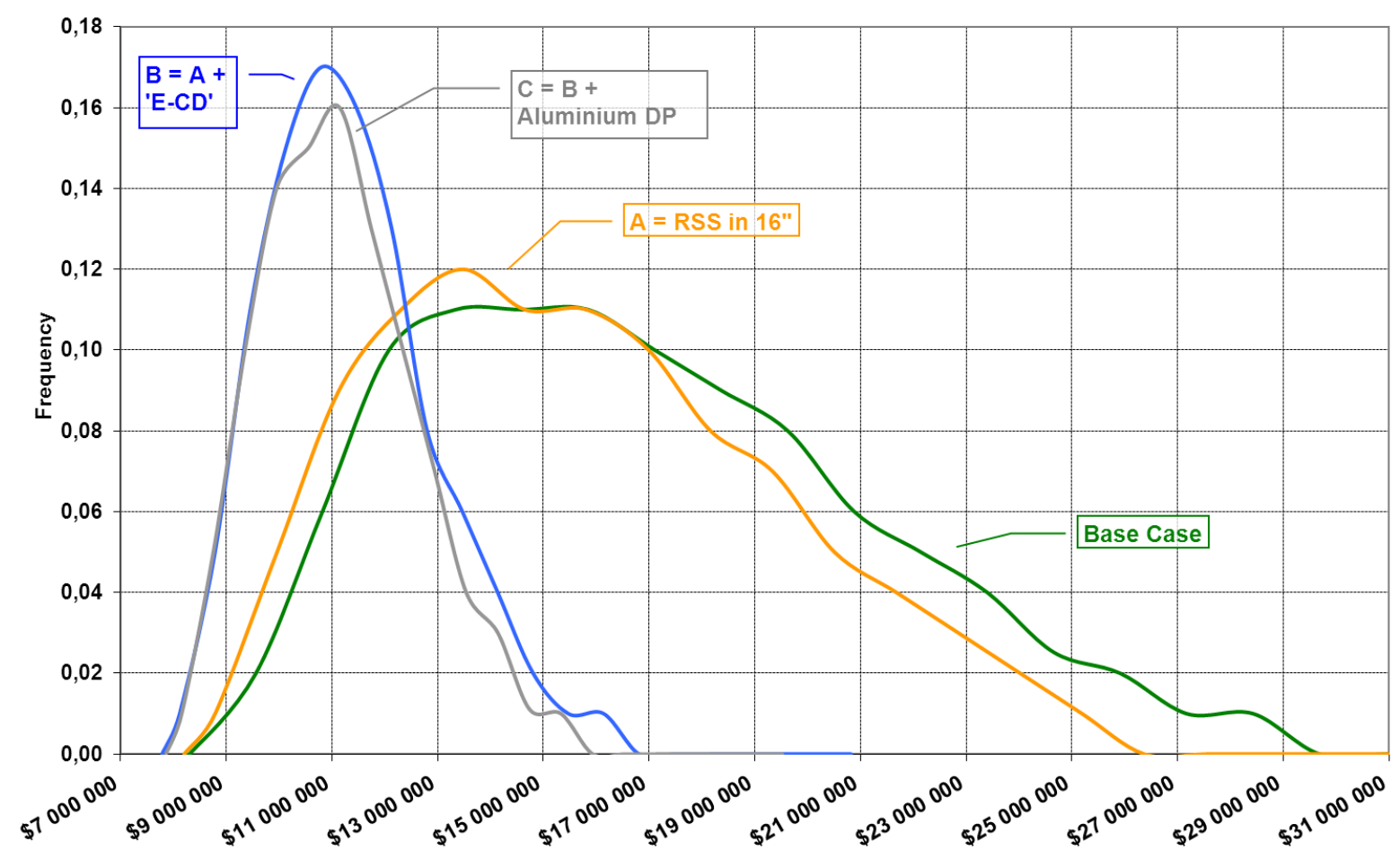

Fig(5) Optimization Scenarios

The above graph shows the expected benefits of the proposed actions, including all their inherent costs. All technologies help to reduce the uncertainty spread up to $32 \%$ at $\mathrm{P} 50$ as shown on the following cumulative curves and table. In particular, in this case, the introduction of the E-CD (scenario B) gave the main benefit in terms of reduction of the uncertainty. 


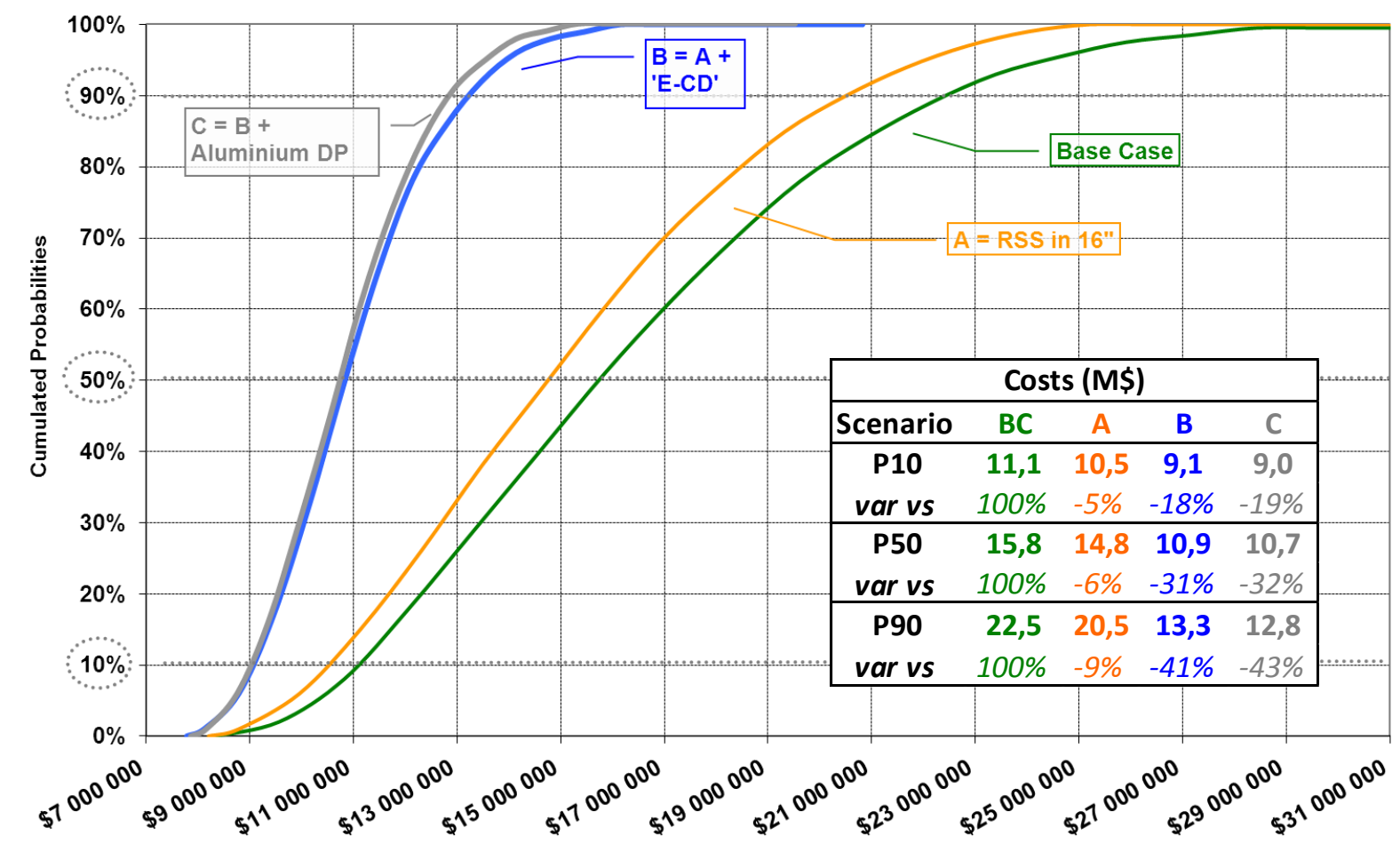

Fig.(6) Scenarios Cumulated Probabilities

Decision was made to drill the well applying all mitigation action provided in the scenario $\mathrm{C}$ that was giving the lowest cost at P50, 10,7 $\mathrm{M} \$$, to be compared to the $21+\mathrm{M} \$$ actual cost of some similar reference wells 1 and 3.

\section{Cost Follow-Up \& Lessons}

\section{Learned}

It is important to highlight that:

- While the project is under way and some activities are completed, variance analysis can be undertaken to compare forecasted cost and duration to the outturn. This is an important analysis as part of the post well process.

- During project execution, any important deviation could require the re-authorization of the AFE. All revised forecasts has to be made on the available probabilistic model. However, this was not necessary for the case under study. 
- The final project outturn is used to update the integrated system used for RM of the technical aspects of future projects. In this way lessons learned in one project provides feedback for future projects and embodies a continuous learning process.

Regarding the case study, the post well analysis is limited to drilling operations, from rig skid to drill 6" hole at $7940 \mathrm{MD}$, in terms of:
- Time versus depth

- Actual versus forecasted costs

- Risks versus NPT

In order to work on a common basis, initial estimates were readjusted with the actual phase lengths.

A series of indicators can be measured in a post well analysis. A standard one is the Time versus depth comparison illustrated and commented on the below graph:

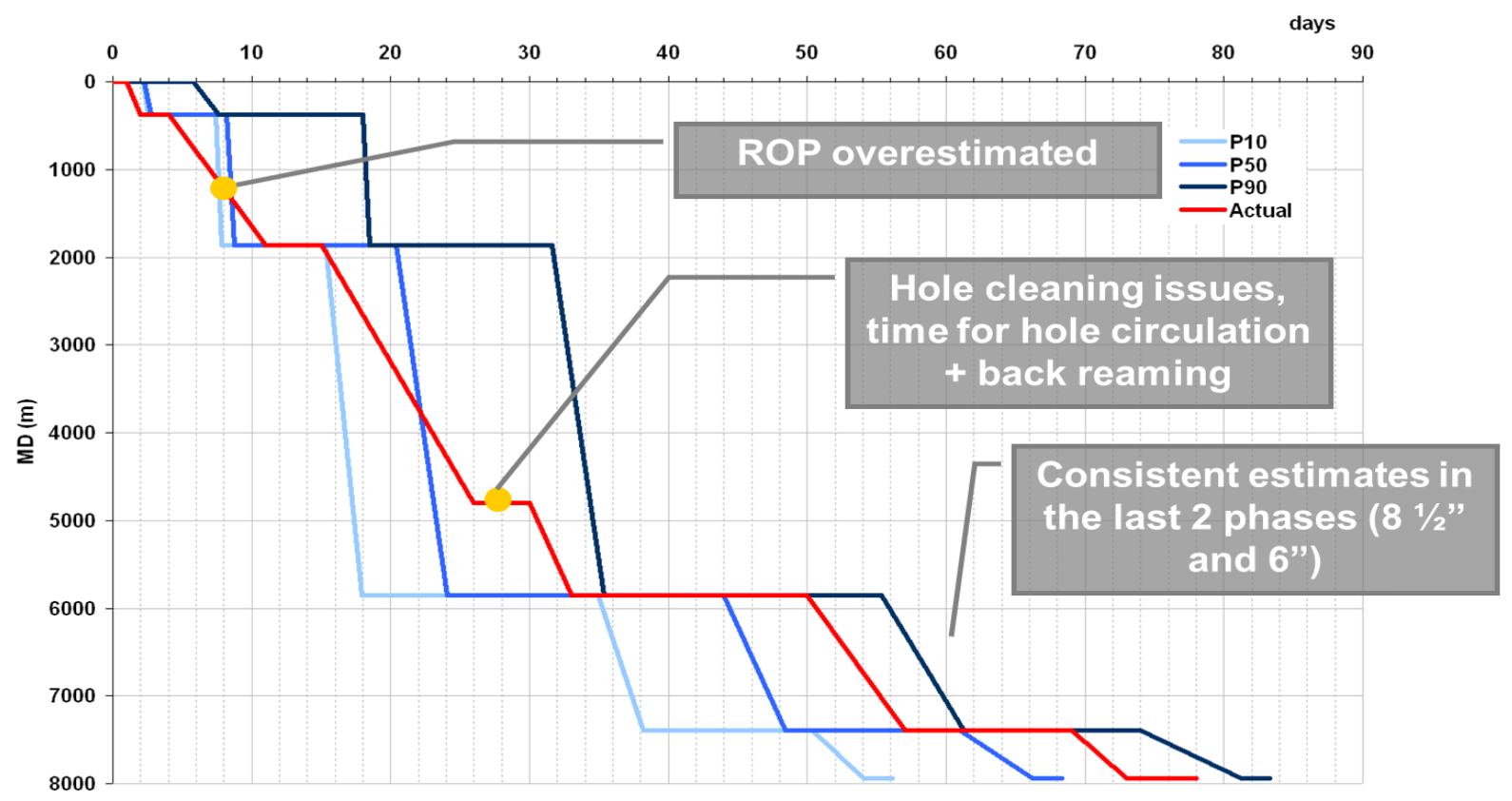

Fig.(7) Times versus Depth Post Well Analysis

The time versus depth indicates discrepancies in the surface and that duration forecast were relevant intermediate phases. These for the last 2 phases, but shows some discrepancies were analyzed on the 
drilling model and the drilling records. It came out that that ROP was overestimated in the surface section and circulation time was underestimated in the intermediate phase.
Regarding the cost, the actual cost was represented on the following estimated cumulative distribution:

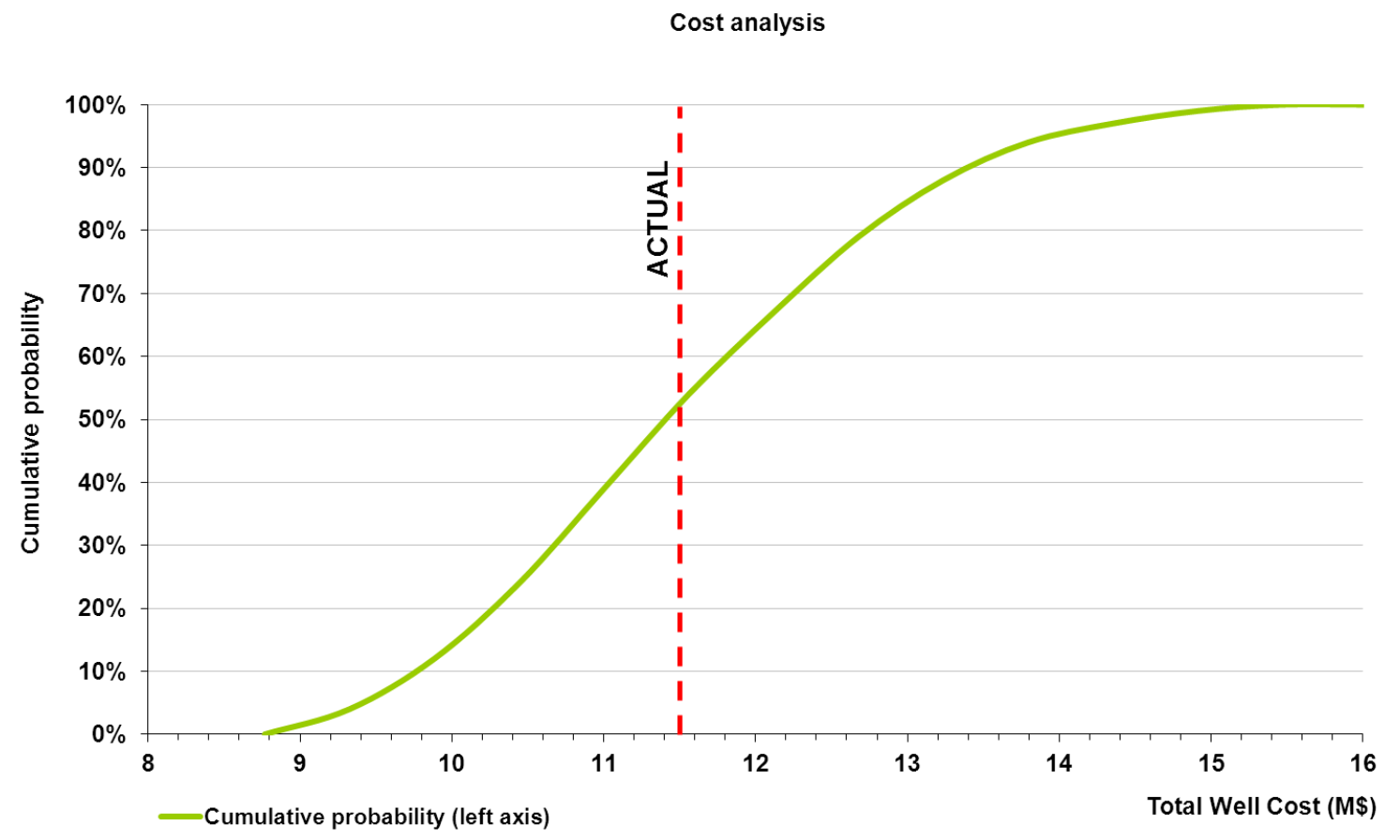

Fig.(8) Planned versus Actual Cost

Actual well cost (at end 6" phase) is close to P50, so it can be concluded that overall estimates presented a good accuracy.

Regarding well problems, a comparison was made between recorded NPT during well execution and the risk identified during project studies. Two graphic representations are presented, one addressing the accuracy of the risk identification and another one addressing the accuracy of the risk evaluation.

Regarding risk identification, 
identified risks are represented in the outer circle of the following graph, while actual NPT are represented in the inner circle. The size of each section represents either the relative impact of each risk on the total impact or the relative weight of each NPT on the total NPT.

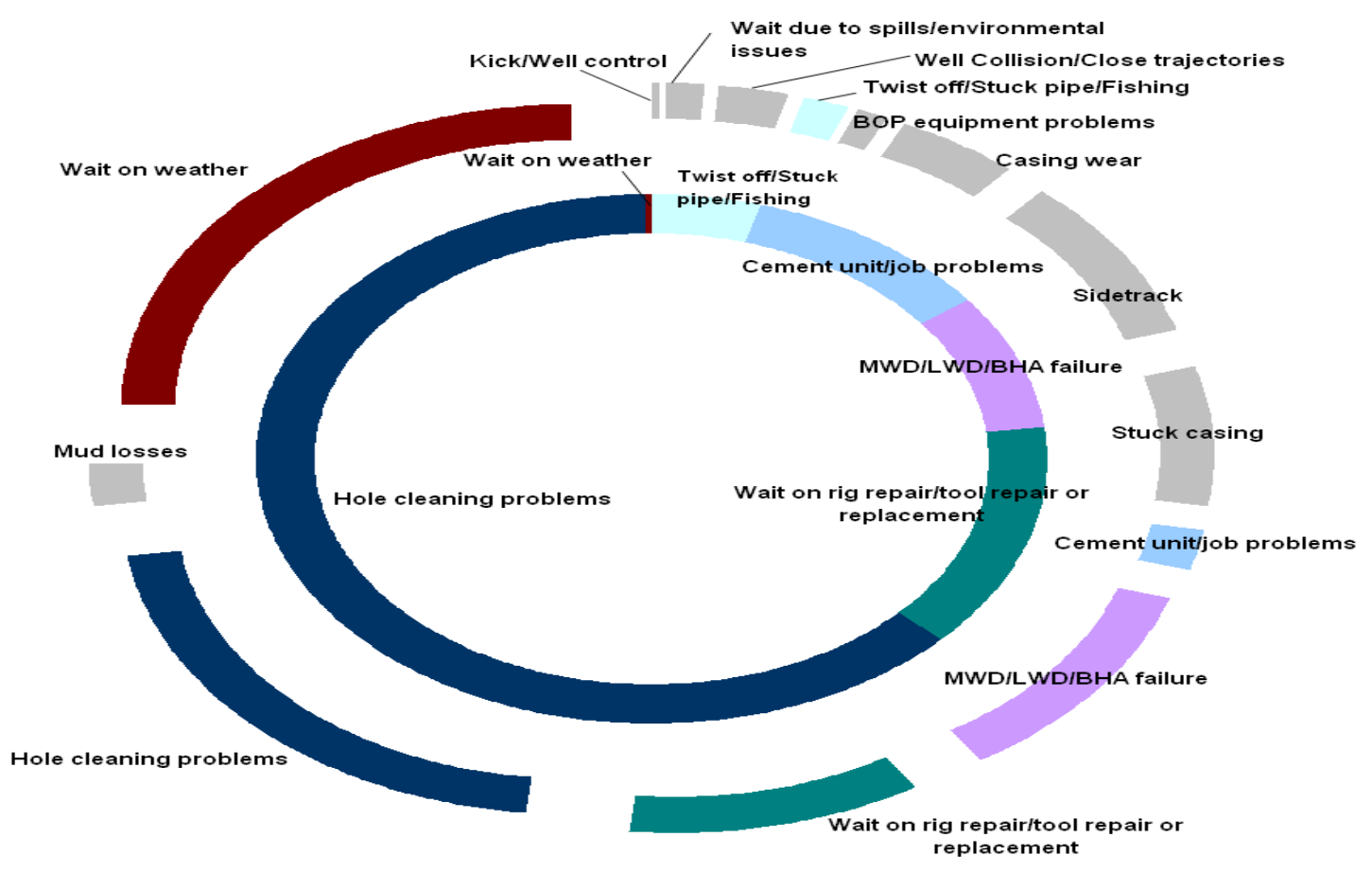

Fig.(9) Risk Identification versus NPT

The first conclusion is that all NPT were addressed in the risk register. However a number of risks did not occur, as illustrated in the risk evaluation versus NPT comparison: 


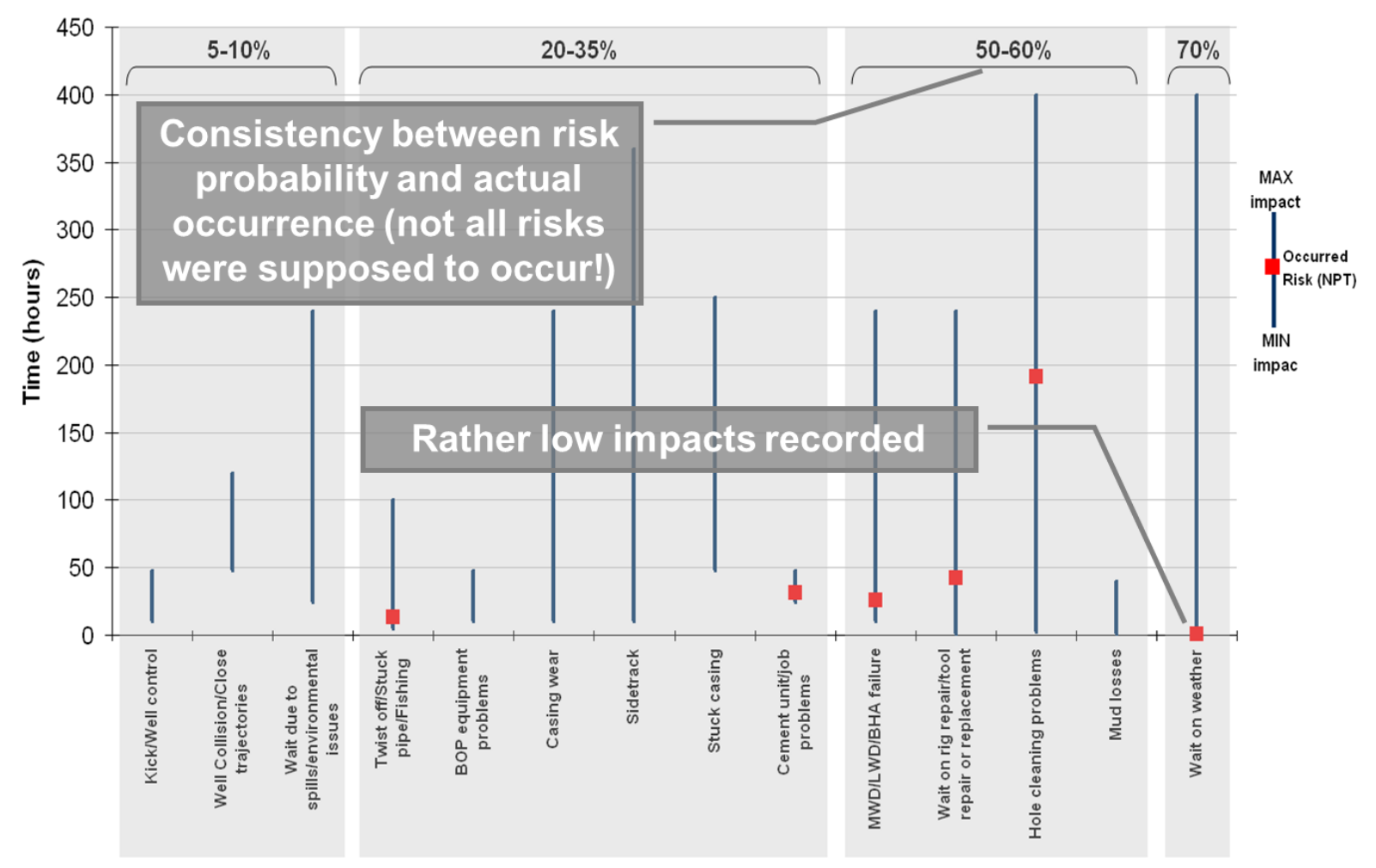

Fig.(10) Risk Evaluation versus NPT

The conclusions regarding the risk assessment activities performed in the case study are:

- The risk evaluation was rather consistent for:

- Hole cleaning issues

- Wait on repair / other

- BHA failure

- Cementing problems

- A number of risks did not occurred:
- Stuck Casing (mitigated by trajectory optimization).

- Sidetrack (mitigated by RSS).

- Casing wear (mitigated by trajectory optimization) .

○ ...other risk issues with low probability of occurrence

- Risk evaluation was inconsistent, and the impact 
was overestimated, for the

following issues:

- Wait on weather (no activities performed during monsoon).

- Stuck Pipe (mitigated by trajectory optimization and ECD).
In this post well analysis the risks seem to be somehow overestimated compared to the recorded NPT, nonetheless the overall cost estimate is consistent with the actual cost of the well. The discrepancy is then in the normal versus trouble time breakdown as show in the following histogram:

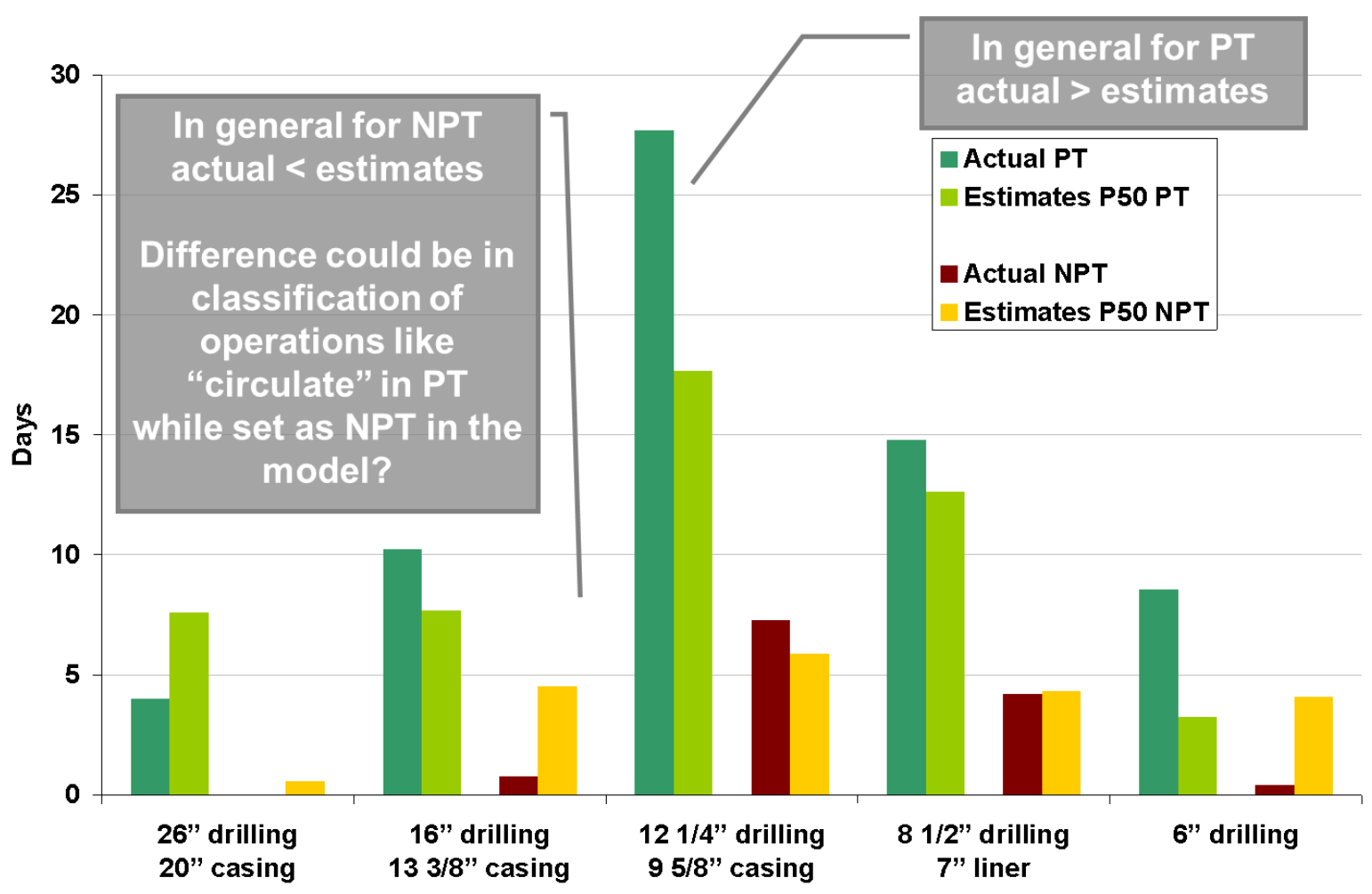

Fig.(11) Normal Time versus Non Productive Time

It is clear that referring to Normal Time, the actual values are higher that the forecast. On the contrary, when referring to the NPT, the actual values are lower than the forecast. This illustrates a tendency to emphasize the attention on the potential problem issues during the planning stage, and to hide 
operational problems in normal operations during operations.

\section{Conclusions}

$\mathrm{RM}$

approach

is

recommended for all D\&C AFE estimates or for any decisional process related to:

- Technologies implementation

- Scenario comparison

- Contingencies calculation

In this case, the cost risk study was efficient in:

- Estimating technologies benefits

- Support cost and time estimates, reducing uncertainty spread in challenging situations

- Giving an accurate value for AFE (P50)

$\mathrm{RM}$ approach added value relies on a series of key factors of success:

- Tailored workflow: provide adequate RM response for each project characteristics (avoid over complexity, be exhaustive).

- Early implementation of RM process in feasibility phase, followed by a progressive deployment.

- Consider both risk \& opportunities.

- Remain compliant with best practices in industry.

\section{Nomenclature}

ADP Aluminium Drill Pipes AFE Authorization For Expenditures

AWARE Advised Workflow for Accurate Risk Estimates.

CAPEX CAPital EXpenditure

D\&C Drilling \& Completion

E-CD Eni Circulation Device

NPT Non Productive Time

OPEX OPeration EXpenditure

RM Risk Management

RSS Rotary Steerable System. 


\section{$\underline{\text { References }}$}

1. A Guide to the Project Risk Management Body of Knowledge (PMBOK Guide), third edition, Chapter 11.

2. S. Masi, C. Molaschi, F. Zausa, J. Michelez, N. Rossi(2010), Key Factors Sensitivity Analysis on Blowout Probability in Dangerous Drilling Conditions Applying Different Technical Solutions. SPE 133027-MS, . 